\title{
Parametrization of flavor mixing in the standard model
}

\author{
Harald Fritzsch* \\ Theory Division, CERN, CH-1211 Geneva 23, Switzerland \\ and Sektion Physik, Universität München, 80333 München, Germany \\ Zhi-zhong Xing ${ }^{\dagger}$ \\ Department of Physics, Nagoya University, Nagoya 464-01, Japan
}

(Received 15 August 1997; published 26 November 1997)

\begin{abstract}
It is shown that there exist nine different ways to describe the flavor mixing, in terms of three rotation angles and one $C P$-violating phase, within the standard electroweak theory of six quarks. For the assignment of the complex phase there essentially exists a continuum of possibilities, if one allows the phase to appear in more than four elements of the mixing matrix. If the phase is restricted to four elements, the phase assignment is uniquely defined. If one imposes the constraint that the phase disappears in a natural way in the chiral limit in which the masses of the $u$ and $d$ quarks are turned off, only three of the nine parametrizations are acceptable. In particular the "standard" parametrization advocated by the Particle Data Group is not permitted. One parametrization, in which the $C P$-violating phase is restricted to the light quark sector, stands up as the most favorable description of the flavor mixing.
\end{abstract}

[S0556-2821(98)00803-0]

PACS number(s): 12.15.Hh, 11.30.Er, 12.15.Ff

In the standard electroweak theory, the phenomenon of flavor mixing of the quarks is described by a $3 \times 3$ unitary matrix, the Cabibbo-Kobayashi-Maskawa (CKM) matrix $[1,2]$. This matrix can be expressed in terms of four parameters, which are usually taken as three rotation angles and one phase. A number of different parametrizations have been proposed in the literature [2-5]. Of course, adopting a particular parametrization of flavor mixing is arbitrary and not directly a physical issue. Nevertheless it is quite likely that the actual values of flavor mixing parameters (including the strength of $C P$ violation), once they are known with high precision, will give interesting information about the physics beyond the standard model. Probably at this point it will turn out that a particular description of the CKM matrix is more useful and transparent than the others. For this reason, we find it useful to analyze all possible parametrizations and to point out their respective advantages and disadvantages. This is the main purpose of this Brief Report.

In the standard model the quark flavor mixing arises once the up- and down-type mass matrices are diagonalized. The generation of quark masses is intimately related to the phenomenon of flavor mixing. In particular, the flavor mixing parameters do depend on the elements of quark mass matrices. A particular structure of the underlying mass matrices calls for a particular choice of the parametrization of the flavor mixing matrix. For example, in Ref. [6] it was noticed that a rather special form of the flavor mixing matrix results if one starts from Hermitian mass matrices in which the $(1,3)$ and $(3,1)$ elements vanish. This has been subsequently observed again in a number of papers [7]. Recently we have studied the exact form of such a description from a general point of view and pointed out some advantages of this type of representation in the discussion of flavor mixing and

\footnotetext{
*Electronic address: bm@hep.physik.uni-muenchen.de

†Electronic address: xing@eken.phys.nagoya-u.ac.jp
}

$C P$-violating phenomena [5]. One of the aims of this work is also to view this parametrization in the context with other ways of describing the flavor mixing.

In the standard model the weak charged currents are given by

$$
\overline{(u, c, t)}{ }_{L}\left(\begin{array}{ccc}
V_{u d} & V_{u s} & V_{u b} \\
V_{c d} & V_{c s} & V_{c b} \\
V_{t d} & V_{t s} & V_{t b}
\end{array}\right)\left(\begin{array}{c}
d \\
s \\
b
\end{array}\right)_{L},
$$

where $u, c, \ldots, b$ are the quark mass eigenstates, $L$ denotes the left-handed fields, and $V_{i j}$ are elements of the CKM matrix $V$. In general $V_{i j}$ are complex numbers, but their absolute values are measurable quantities. For example, $\left|V_{c b}\right|$ primarily determines the lifetime of $B$ mesons. The phases of $V_{i j}$, however, are not physical, like the phases of quark fields. A phase transformation of the $u$ quark $\left(u \rightarrow u e^{\mathrm{i} \alpha}\right)$, for example, leaves the quark mass term invariant but changes the elements in the first row of $V$ (i.e., $V_{u j} \rightarrow V_{u j} e^{-\mathrm{i} \alpha}$ ). Only a common phase transformation of all quark fields leaves all elements of $V$ invariant, thus there is a fivefold freedom to adjust the phases of $V_{i j}$.

In general the unitary matrix $V$ depends on nine parameters. Note that in the absence of complex phases $V$ would consist of only three independent parameters, corresponding to three (Euler) rotation angles. Hence one can describe the complex matrix $V$ by three angles and six phases. Due to the freedom in redefining the quark field phases, five of the six phases in $V$ can be absorbed; and we arrive at the wellknown result that the CKM matrix $V$ can be parametrized in terms of three rotation angles and one $C P$-violating phase. The question about how many different ways to describe $V$ may exist was raised some time ago [8]. Below we shall reconsider this problem and give a complete analysis.

If the flavor mixing matrix $V$ is first assumed to be a real orthogonal matrix, it can in general be written as a product of three matrices $R_{12}, R_{23}$ and $R_{31}$, which describe simple rotations in the $(1,2),(2,3)$ and $(3,1)$ planes: 


$$
\begin{aligned}
R_{12}(\theta) & =\left(\begin{array}{ccc}
c_{\theta} & s_{\theta} & 0 \\
-s_{\theta} & c_{\theta} & 0 \\
0 & 0 & 1
\end{array}\right), \\
R_{23}(\sigma) & =\left(\begin{array}{ccc}
1 & 0 & 0 \\
0 & c_{\sigma} & s_{\sigma} \\
0 & -s_{\sigma} & c_{\sigma}
\end{array}\right), \\
R_{31}(\tau) & =\left(\begin{array}{ccc}
c_{\tau} & 0 & s_{\tau} \\
0 & 1 & 0 \\
-s_{\tau} & 0 & c_{\tau}
\end{array}\right),
\end{aligned}
$$

where $s_{\theta} \equiv \sin \theta, c_{\theta} \equiv \cos \theta$, etc. Clearly any two rotation matrices do not commute with each other. There exist twelve different ways to arrange products of these matrices such that the most general orthogonal matrix $R$ can be obtained [8]. Note that the matrix $R_{i j}^{-1}(\omega)$ plays an equivalent role as $R_{i j}(\omega)$ in constructing $R$, because of $R_{i j}^{-1}(\omega)=R_{i j}(-\omega)$. Note also that $R_{i j}(\omega) R_{i j}\left(\omega^{\prime}\right)=R_{i j}\left(\omega+\omega^{\prime}\right)$ holds, thus the product $R_{i j}(\omega) R_{i j}\left(\omega^{\prime}\right) R_{k l}\left(\omega^{\prime \prime}\right)$ or $R_{k l}\left(\omega^{\prime \prime}\right) R_{i j}(\omega) R_{i j}\left(\omega^{\prime}\right)$ cannot cover the whole space of a $3 \times 3$ orthogonal matrix and should be excluded. Explicitly the twelve different forms of $R$ read as

$$
\begin{aligned}
& \text { (1) } \quad R=R_{12}(\theta) R_{23}(\sigma) R_{12}\left(\theta^{\prime}\right), \\
& \text { (2) } \quad R=R_{12}(\theta) R_{31}(\tau) R_{12}\left(\theta^{\prime}\right), \\
& \text { (3) } \quad R=R_{23}(\sigma) R_{12}(\theta) R_{23}\left(\sigma^{\prime}\right), \\
& \text { (4) } \quad R=R_{23}(\sigma) R_{31}(\tau) R_{23}\left(\sigma^{\prime}\right), \\
& \text { (5) } \quad R=R_{31}(\tau) R_{12}(\theta) R_{31}\left(\tau^{\prime}\right), \\
& \text { (6) } \quad R=R_{31}(\tau) R_{23}(\sigma) R_{31}\left(\tau^{\prime}\right),
\end{aligned}
$$

in which a rotation in the $(i, j)$ plane occurs twice; and

$$
\begin{aligned}
& \text { (7) } \quad R=R_{12}(\theta) R_{23}(\sigma) R_{31}(\tau), \\
& \text { (8) } \quad R=R_{12}(\theta) R_{31}(\tau) R_{23}(\sigma), \\
& \text { (9) } \quad R=R_{23}(\sigma) R_{12}(\theta) R_{31}(\tau), \\
& \text { (10) } \quad R=R_{23}(\sigma) R_{31}(\tau) R_{12}(\theta), \\
& \text { (11) } \quad R=R_{31}(\tau) R_{12}(\theta) R_{23}(\sigma), \\
& \text { (12) } \quad R=R_{31}(\tau) R_{23}(\sigma) R_{12}(\theta),
\end{aligned}
$$

where all three $R_{i j}$ are present.

Although all the above twelve combinations represent the most general orthogonal matrices, only nine of them are structurally different. The reason is that the products $R_{i j} R_{k l} R_{i j}$ and $R_{i j} R_{m n} R_{i j}$ (with $i j \neq k l \neq m n$ ) are correlated with each other, leading essentially to the same form for $R$. Indeed it is straightforward to see the correlation between patterns (1), (3), (5) and (2), (4), (6), respectively, as follows:

$$
\begin{aligned}
R_{12}(\theta) R_{31}(\tau) R_{12}\left(\theta^{\prime}\right)= & R_{12}(\theta+\pi / 2) R_{23}(\sigma=\tau) \\
& \times R_{12}\left(\theta^{\prime}-\pi / 2\right), \\
R_{23}(\sigma) R_{31}(\tau) R_{23}\left(\sigma^{\prime}\right)= & R_{23}(\sigma-\pi / 2) R_{12}(\theta=\tau) \\
& \times R_{23}\left(\sigma^{\prime}+\pi / 2\right), \\
R_{31}(\tau) R_{23}(\sigma) R_{31}\left(\tau^{\prime}\right)= & R_{31}(\tau+\pi / 2) R_{12}(\theta=\sigma) \\
& \times R_{31}\left(\tau^{\prime}-\pi / 2\right) .
\end{aligned}
$$

Thus the orthogonal matrices (2), (4) and (6) need not be treated as independent choices. We then draw the conclusion that there exist nine different forms for the orthogonal matrix $R$, i.e., patterns (1), (3) and (5) as well as (7)-(12).

We proceed to include the $C P$-violating phase, denoted by $\varphi$, in the above rotation matrices. The resultant matrices should be unitary such that a unitary flavor mixing matrix can be finally produced. There are several different ways for $\varphi$ to enter $R_{12}$, e.g.,

$$
R_{12}(\theta, \varphi)=\left(\begin{array}{ccc}
c_{\theta} & s_{\theta} e^{+\mathrm{i} \varphi} & 0 \\
-s_{\theta} e^{-\mathrm{i} \varphi} & c_{\theta} & 0 \\
0 & 0 & 1
\end{array}\right)
$$

or

$$
R_{12}(\theta, \varphi)=\left(\begin{array}{ccc}
c_{\theta} & s_{\theta} & 0 \\
-s_{\theta} & c_{\theta} & 0 \\
0 & 0 & e^{-\mathrm{i} \varphi}
\end{array}\right)
$$

or

$$
R_{12}(\theta, \varphi)=\left(\begin{array}{ccc}
c_{\theta} e^{+\mathrm{i} \varphi} & s_{\theta} & 0 \\
-s_{\theta} & c_{\theta} e^{-\mathrm{i} \varphi} & 0 \\
0 & 0 & 1
\end{array}\right)
$$

Similarly one may introduce a phase parameter into $R_{23}$ or $R_{31}$. Then the CKM matrix $V$ can be constructed, as a product of three rotation matrices, by use of one complex $R_{i j}$ and two real ones. Note that the location of the $C P$-violating phase in $V$ can be arranged by redefining the quark field phases, thus it does not play an essential role in classifying different parametrizations. We find that it is always possible to locate the phase parameter $\varphi$ in a $2 \times 2$ submatrix of $V$, in which each element is a sum of two terms with the relative phase $\varphi$. The remaining five elements of $V$ are real in such a phase assignment. Accordingly we arrive at nine distinctive parametrizations of the CKM matrix $V$, as listed in Table I, where the complex rotation matrices $R_{12}(\theta, \varphi), R_{23}(\sigma, \varphi)$ and $R_{31}(\tau, \varphi)$ are obtained directly from the real ones in Eq. (2) with the replacement $1 \rightarrow e^{-i \varphi}$.

Some instructive relations of each parametrization, as well as the rephasing-invariant measure of $C P$ violation [9] defined by $\mathcal{J}$ through

$$
\operatorname{Im}\left(V_{i l} V_{j m} V_{i m}^{*} V_{j l}^{*}\right)=\mathcal{J} \sum_{k, n=1}^{3}\left(\epsilon_{i j k} \epsilon_{l m n}\right),
$$


TABLE I. Classification of different parametrizations for the flavor mixing matrix.

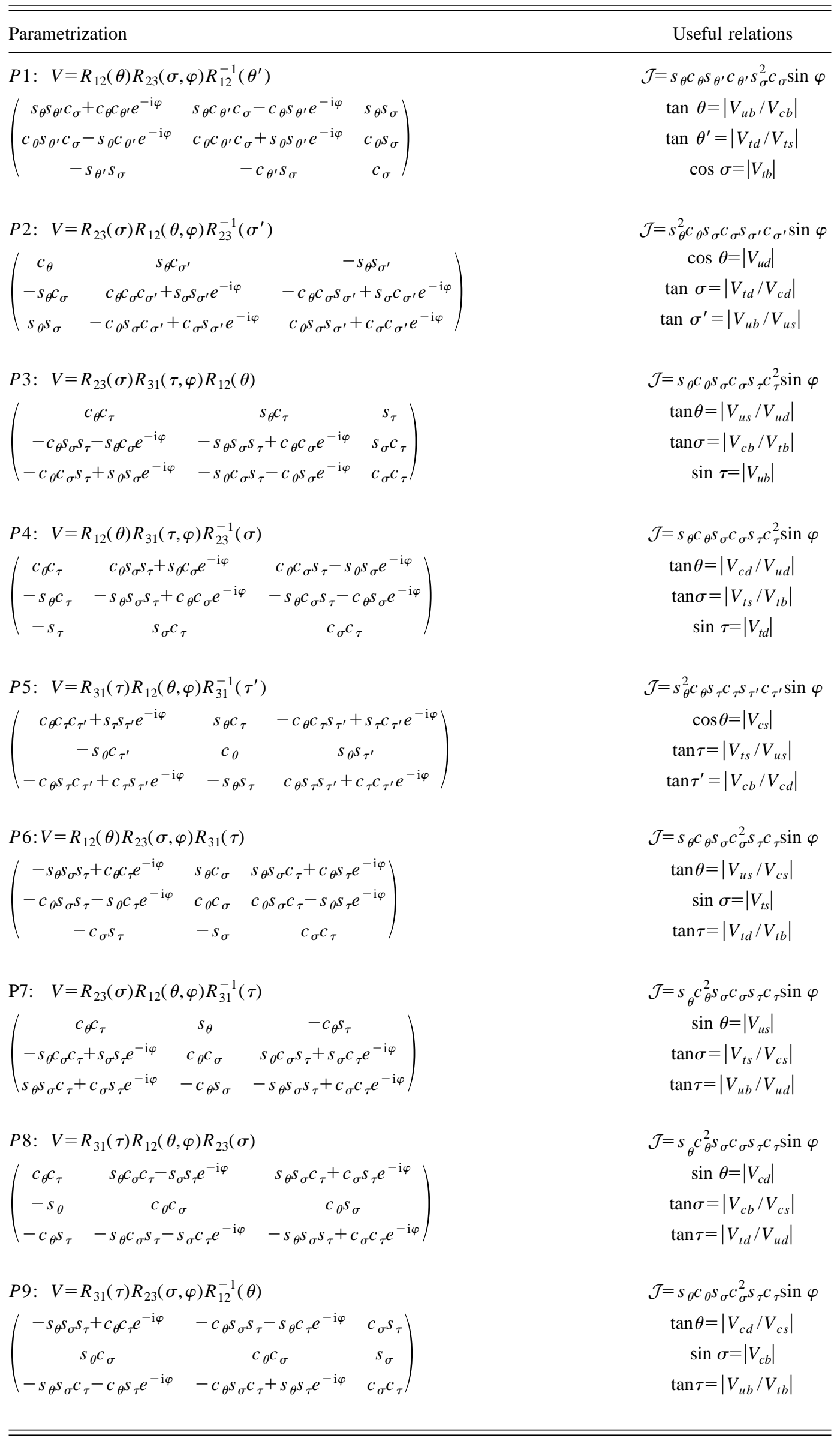


have also been given in Table I. One can see that $P 2$ and $P 3$ correspond to the Kobayashi-Maskawa [2] and Maiani [3] representations, although different notations for the $C P$-violating phase and three mixing angles are adopted here. The latter is indeed equivalent to the "standard" parametrization advocated by the Particle Data Group $[3,4]$. This can be seen clearly if one makes three transformations of quark field phases: $c \rightarrow c e^{-\mathrm{i} \varphi}, t \rightarrow t e^{-\mathrm{i} \varphi}$, and $b \rightarrow b e^{-\mathrm{i} \varphi}$. In addition, $P 1$ is just the one proposed by the present authors in Ref. [5].

From a mathematical point of view, all nine different parametrizations are equivalent. However this is not the case if we apply our considerations to the quarks and their mass spectrum. It is well known that both the observed quark mass spectrum and the observed values of the flavor mixing parameters exhibit a striking hierarchical structure. The latter can be understood in a natural way as the consequence of a specific pattern of chiral symmetries whose breaking causes the towers of different masses to appear step by step [1012]. Such a chiral evolution of the mass matrices leads, as argued in Ref. [11], to a specific way to introduce and describe the flavor mixing. In the limit $m_{u}=m_{d}=0$, which is close to the real world, since $m_{u} / m_{t} \ll 1$ and $m_{d} / m_{b} \ll 1$, the flavor mixing is merely a rotation between the $t-c$ and $b-s$ systems, described by one rotation angle. No complex phase is present; i.e., $C P$ violation is absent. This rotation angle is expected to change very little, once $m_{u}$ and $m_{d}$ are introduced as tiny perturbations. A sensible parametrization should make use of this feature. This implies that the rotation matrix $R_{23}$ appears exactly once in the description of the CKM matrix $V$, eliminating $P 2$ (in which $R_{23}$ appears twice) and $P 5$ (where $R_{23}$ is absent). This leaves us with seven parametrizations of the flavor mixing matrix.

The list can be reduced further by considering the location of the phase $\varphi$. In the limit $m_{u}=m_{d}=0$, the phase must disappear in the weak transition elements $V_{t b}, V_{t s}, V_{c b}$ and $V_{c s}$. In $P 7$ and $P 8$, however, $\varphi$ appears particularly in $V_{t b}$. Thus these two parametrizations should be eliminated, leaving us with five parametrizations (i.e., $P 1, P 3, P 4, P 6$ and $P 9)$. In the same limit, the phase $\varphi$ appears in the $V_{t s}$ element of $P 3$ and the $V_{c b}$ element of $P 4$. Hence these two parametrizations should also be eliminated. Then we are left with three parametrizations, $P 1, P 6$ and $P 9$. As expected, these are the parametrizations containing the complex rotation matrix $R_{23}(\sigma, \varphi)$. We stress that the "standard" parametrization [4] (equivalent to $P 3$ ) does not obey the above constraints and should be dismissed.

Among the remaining three parametrizations, $P 1$ is singled out by the fact that the $C P$-violating phase $\varphi$ appears only in the $2 \times 2$ submatrix of $V$ describing the weak transitions among the light quarks. This is precisely the system where the phase $\varphi$ should appear, not in any of the weak transition elements involving the heavy quarks $t$ and $b$.

In the parametrization $P 6$ or $P 9$, the complex phase $\varphi$ appears in $V_{c b}$ or $V_{t s}$, but this phase factor is multiplied by a product of $\sin \theta$ and $\sin \tau$, i.e., it is of second order of the weak mixing angles. Hence the imaginary parts of these elements are not exactly vanishing, but very small in magnitude.

In our view the best possibility to describe the flavor mixing in the standard model is to adopt the parametrization $P 1$. As discussed in Ref. [5], this parametrization has a number of significant advantages in addition to that mentioned above. Especially it is well suited for specific models of quark mass matrices (see, e.g., Refs. [6,7]).

We conclude: there are nine different ways to describe a real $3 \times 3$ flavor mixing matrix in terms of three rotation angles. Introducing a complex phase $\varphi$ does not increase the number of distinct parametrizations, except for the fact that there is a continuum of possibilities for assigning the phase factors. Imposing natural constraints in view of the observed mass hierarchy [i.e., in the limit $m_{u}=m_{d}=0$ phases should be absent in the $(2,2),(2,3),(3,2)$ and $(3,3)$ elements of the mixing matrix], we can eliminate six parametrizations, including the original Kobayashi-Maskawa parametrization [2] and the "standard" parametrization proposed in Refs. [3,4]. We propose to use the parametrization $P 1$ for the further study of flavor mixing and $C P$-violating phenomena.

Note added After completion of this work we received a report of Rasin [13], in which part of the conclusions drawn here was also reached.

The work of H.F. was supported in part by GIF contract I-0304-120.07/93 and EEC contract CHRX-CT94-0579 (DG 12 COMA). Z.Z.X. is grateful to A.I. Sanda for his warm hospitality and to the Japan Society for the Promotion of Science for its financial support.
[1] N. Cabibbo, Phys. Rev. Lett. 10, 531 (1963).

[2] M. Kobayashi and T. Maskawa, Prog. Theor. Phys. 49, 652 (1973).

[3] See, e.g., L. Maiani, Phys. Lett. 62B, 183 (1976); L. L. Chau and W. Y. Keung, Phys. Rev. Lett. 53, 1802 (1984); H. Fritzsch, Phys. Rev. D 32, 3058 (1985); M. Gironau and J. Schechter, Phys. Rev. Lett. 54, 385 (1985); H. Harari and M. Leurer, Phys. Lett. B 181, 123 (1986); H. Fritzsch and J. Plankl, Phys. Rev. D 35, 1732 (1987).

[4] F. J. Gilman, K. Kleinknecht, and B. Renk, Phys. Rev. D 54, 94 (1996).

[5] H. Fritzsch and Z. Z. Xing, Report No. YITP-97-31 or hep-ph/9707215 [Phys. Lett. B (to be published)].

[6] H. Fritzsch, Nucl. Phys. B155, 189 (1979).
[7] S. Dimopoulos, L. J. Hall, and S. Raby, Phys. Rev. D 45, 4192 (1992); L. J. Hall and A. Rasin, Phys. Lett. B 315, 164 (1993); H. Lehmann, C. Newton, and T. T. Wu, ibid. 384, 249 (1996); R. Barbieri, L. J. Hall, and A. Romanino, ibid. 401, 47 (1997).

[8] C. Jarlskog, in CP Violation, edited by C. Jarlskog (World Scientific, Singapore, 1989), p. 3.

[9] C. Jarlskog, Phys. Rev. Lett. 55, 1039 (1985); D. D. Wu, Phys. Rev. D 33, 860 (1986).

[10] H. Fritzsch, Phys. Lett. B 184, 391 (1987).

[11] H. Fritzsch, Phys. Lett. B 189, 191 (1987).

[12] L. J. Hall and S. Weinberg, Phys. Rev. D 48, 979 (1993).

[13] A. Rasin, Report No. hep-ph/9708216. 